\section{A systematic review of the characteristics of interventions that promote physical activity in adults with asthma}

Journal of Health Psychology $\mathrm{I}-20$

(C) The Author(s) 2021

\section{(c) (i) \&}

Article reuse guidelines:

sagepub.com/journals-permissions DOI: $10.1177 / 13591053211059386$ journals.sagepub.com/home/hpq

@SAGE

\author{
Leanne Tyson' (iD), Wendy Hardeman', \\ Malcolm Marquette', Joanna Semlyen', \\ Gareth Stratton ${ }^{3}$ and Andrew M Wilson'
}

\begin{abstract}
Physical activity is promoted in the asthma population through pulmonary rehabilitation, but limited funding and facilities are available. This review aimed to examine the effectiveness of interventions that promote physical activity and identify the behaviour change techniques (BCTs) and other intervention components used. Five databases were searched, and 25 studies met the inclusion criteria. Interventions had a significant positive effect on physical activity, sedentary behaviour, quality of life and asthma symptoms. BCTs used across intervention and control groups were similar in studies that showed effects and those that did not. Future interventions should employ techniques that help to maintain behaviour change.
\end{abstract}

\title{
Keywords
}

asthma, physical activity, exercise, intervention, systematic review

\section{Background}

Physical activity is widely recommended in national and international guidelines for asthma management (British Thoracic Society, 2019; Global Initiative for Asthma, 2018). Engaging in regular physical activity ( $\geqslant 150$ minutes/ week of moderate-vigorous physical activity) has shown to have extensive benefits for people living with asthma. Recent reviews have shown that increased physical activity is positively associated with improved lung function, asthma control, health status, and healthcare utilisation (Cordova-Rivera et al., 2018; Hansen et al., 2020). However, despite the guidelines, population-based studies have shown that people living with asthma engage in less physical activity and are more sedentary than people without asthma (van 't Hul et al., 2016).

Pulmonary Rehabilitation (PR) is a comprehensive intervention designed to promote physical activity in patients with respiratory diseases and is defined as 'patient-tailored therapies that

\footnotetext{
'University of East Anglia, UK

${ }^{2}$ Norfolk and Norwich University Hospital, NHS

Foundation Trust, UK

${ }^{3}$ Swansea University, UK

Corresponding author:

Leanne Tyson, Floor 2, Bob Champion Research and

Education Building, James Watson Road, Norwich Medical School, University of East Anglia, Norwich Research Park, Norwich, Norfolk NR4 7UQ, UK.

Email: Leanne.Tyson@uea.ac.uk
} 
include, but are not limited to, exercise training, education, and behaviour change, designed to improve the physical and psychological condition of people with chronic respiratory diseases and to promote the long-term adherence to health-enhancing behaviours' (Spruit et al., 2013:14). PR programmes are usually implemented by a dedicated multi-disciplinary team of healthcare professionals within hospitals or community settings (Spruit et al., 2013). The benefits of PR are well established, including significant positive effects on quality of life and exercise capacity in chronic obstructive pulmonary disease (Jácome and Marques, 2014; McCarthy et al., 2015). However, as asthma patients are increasingly being referred to the programme, systematic reviews have concluded that PR improves quality of life, exercise tolerance, symptoms, reduces the number of exacerbations and could improve pulmonary functions (Feng et al., 2021; Linhas et al., 2017).

Despite the strong evidence and the guidelines recommending the use of PR, patients are significantly under-referred, and of those patients who are referred, uptake and completion are low (National COPD Audit Programme, 2015). A retrospective analysis found that of 711 patients invited to attend PR, 31.8\% did not attend, and a further $29.1 \%$ were non-adherent (Hayton et al., 2013). Major barriers to uptake and completion of PR include travel to attend sessions, disruption to routines, inconvenient timing (Jones et al., 2017), and not being suitable or accessible to those with co-morbidities (Keating et al., 2011). In addition, the coronavirus (COVID-19) and the response to the pandemic (e.g. lockdown and shielding) represent another barrier for patients.

Consequently, there is a need to develop interventions that overcome the major barriers reported by patients to promote physical activity within the asthma population. The benefits of PR are well established, but no systematic reviews to date have examined the effectiveness of other interventions that have been developed to promote physical activity or their components. For instance, there is a lack of evidence regarding the behaviour change techniques
(BCTs), the 'active ingredients' of behaviour change interventions, used within them. BCTs that have been found to be effective in the promotion of physical activity include techniques in the groupings 'Goals and Planning' and 'Feedback and Monitoring' categories, as well as 'Prompts and Cues', 'Graded Tasks' and 'Behavioural Practice/Rehearsal' (Howlett et al., 2019; Samdal et al., 2017). Therefore, the purpose of this review was to examine the effects of interventions that promote physical activity other than formal PR programmes on behavioural and health outcomes in adults diagnosed with asthma and to identify the BCTs and other intervention components, such as intensity, intervention provider, delivery focus, and mode of delivery used within them.

\section{Methods}

The systematic review was conducted in accordance with the Cochrane Handbook for Systematic Reviews of Interventions (Higgins et al., 2021) and the Preferred Reporting for Systematic Reviews and Meta-Analyses (PRISMA) checklist (Moher et al., 2009) (Supplementary File 1). The review protocol was registered on the PROSPERO international prospective register of systematic reviews (CRD42019124032).

\section{Eligibility criteria}

We included published randomised, non-randomised, quasi-experimental, before-and-after interventional studies and feasibility studies. Included interventions had to be designed to promote physical activity and assess relevant behavioural and/or health outcomes, including physical activity, sedentary behaviour, quality of life, asthma control, asthma symptoms and medication usage. Participants had to be aged 18-years or over and have a diagnosis of asthma (any degree of severity). We excluded interventions using PR, defined as exercise training, education, and behaviour change, delivered by a multi-disciplinary team of physicians and healthcare professionals. Having a comparator group was not a requirement for inclusion. 


\section{Data sources}

Comprehensive searches were conducted in MEDLINE, EMBASE, PsycINFO, SPORTDiscus, and The Cochrane Central Register for Clinical Trials. The search strategy (Supplementary File 2) was used to search MEDLINE and modified for other databases. We imposed limits of adults and research papers published in the English language when carrying out the electronic searching, with date restrictions of 1990 and onwards. The search was conducted in August 2020.

\section{Data collection}

Study selection. Studies identified using the searchers were transferred into Mendeley (Mendeley Desktop Version: 1.19.8), and duplicates were removed. Two review authors (LT, MM) independently screened the titles and abstracts of identified studies. The full-text copies of all studies judged to be potentially eligible were then retrieved and screened by the same two reviewers. In case of disagreement, the reviewers reached a consensus through discussion, and reasons for exclusion were recorded (Supplementary File 3).

Data extraction. Data was extracted by the first author (LT) using a standardised data extraction form designed to capture all relevant information, including general study characteristics, characteristics of study participants, details of the intervention and control group components, and study outcomes. Data extraction was independently validated by a second reviewer (MM). Extraction of the BCTs was undertaken by the same two reviewers (LT, MM) using the Behaviour Change Technique Taxonomy v1 (BCTTv1) (Michie et al., 2013) based on the published manuscript and supplementary materials. Both reviewers completed online BCTTv1 training before extraction [http://www.bct-taxonomy.com]. In case of disagreement, a consensus was reached through discussion, and a third reviewer $(\mathrm{WH})$ was involved when the discussion did not lead to a consensus. BCTs were extracted following BCTTv1 guidance, and they were coded as definitely (coded ++ ) or probably (coded + ) present. BCTs were coded in relation to the target behaviours: physical activity and sedentary behaviour.

Risk of bias. The first author (LT) assessed risk of bias of the included studies using the criteria in the Cochrane Handbook for Systematic Reviews of Interventions (Higgins et al., 2021) and assigned judgement of low, high, and unclear. Risk of bias was independently validated by a second reviewer (MM).

\section{Synthesis of results}

Due to the range of very different studies included in this systematic review in relation to the research design, types of intervention and outcome data, the synthesis of the included studies was narrative. Results from the studies were tabulated and organised into groups based on outcomes assessed, so patterns across the data set could be identified. Interventions were deemed effective if they reported significant positive results in all the relevant outcomes they assessed.

\section{Data sharing statement}

The current article includes the data extraction forms for all included studies. Pending acceptance for publication, all the files will be automatically uploaded to the Figshare repository.

\section{Results}

The search yielded 3685 citations, resulting in a total of 2997 after the duplicates were removed. From this list, 92 were identified as potentially relevant, and full texts were retrieved for closer inspection. Two review authors (LT, MM) independently decided that 25 of these articles fulfilled the review's inclusion criteria. Supplementary File 4 shows the detailed process of study inclusion.

\section{Overview of included studies}

The 25 included studies (Table 1) were published between 1992 and 2021. Most studies 


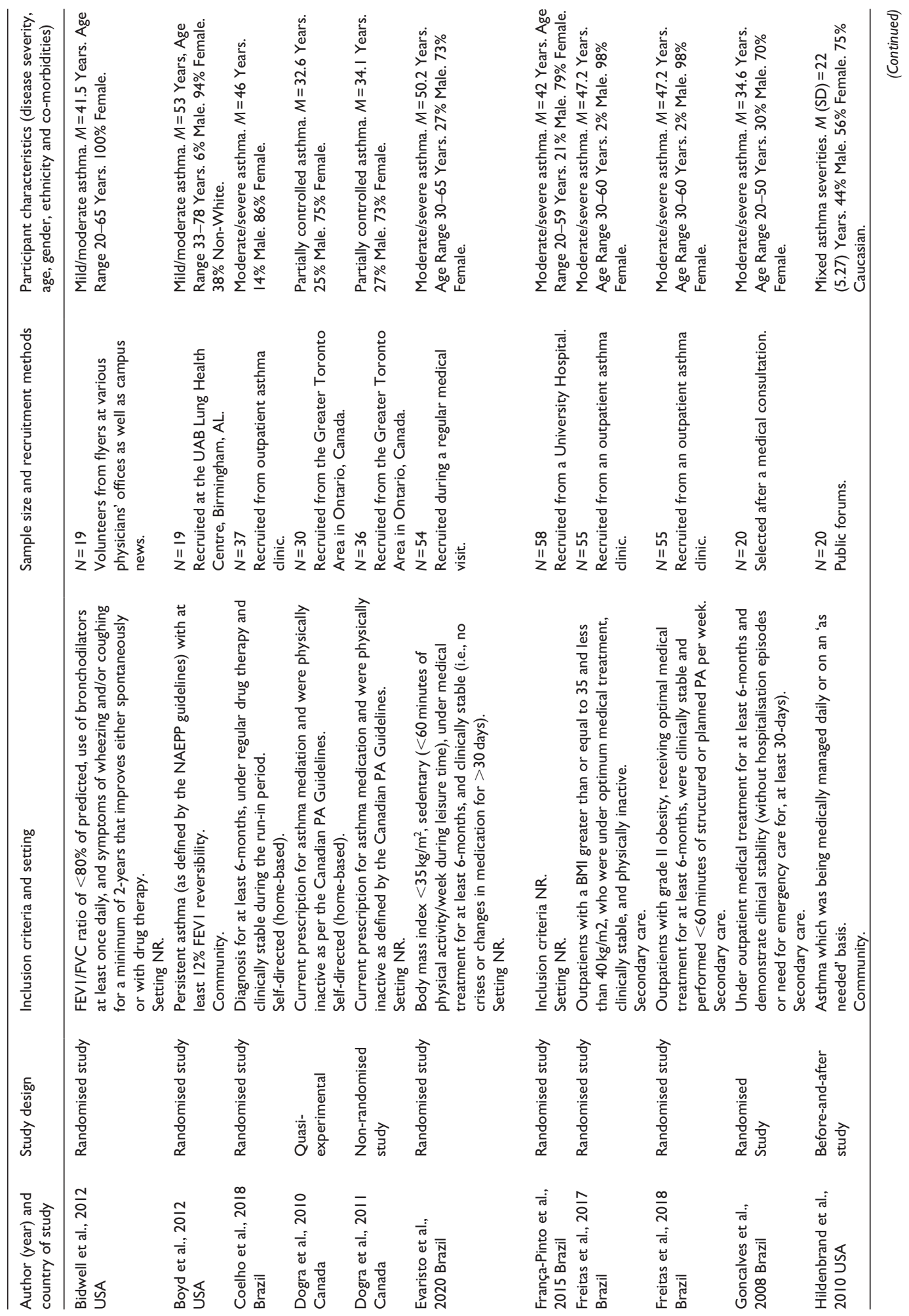




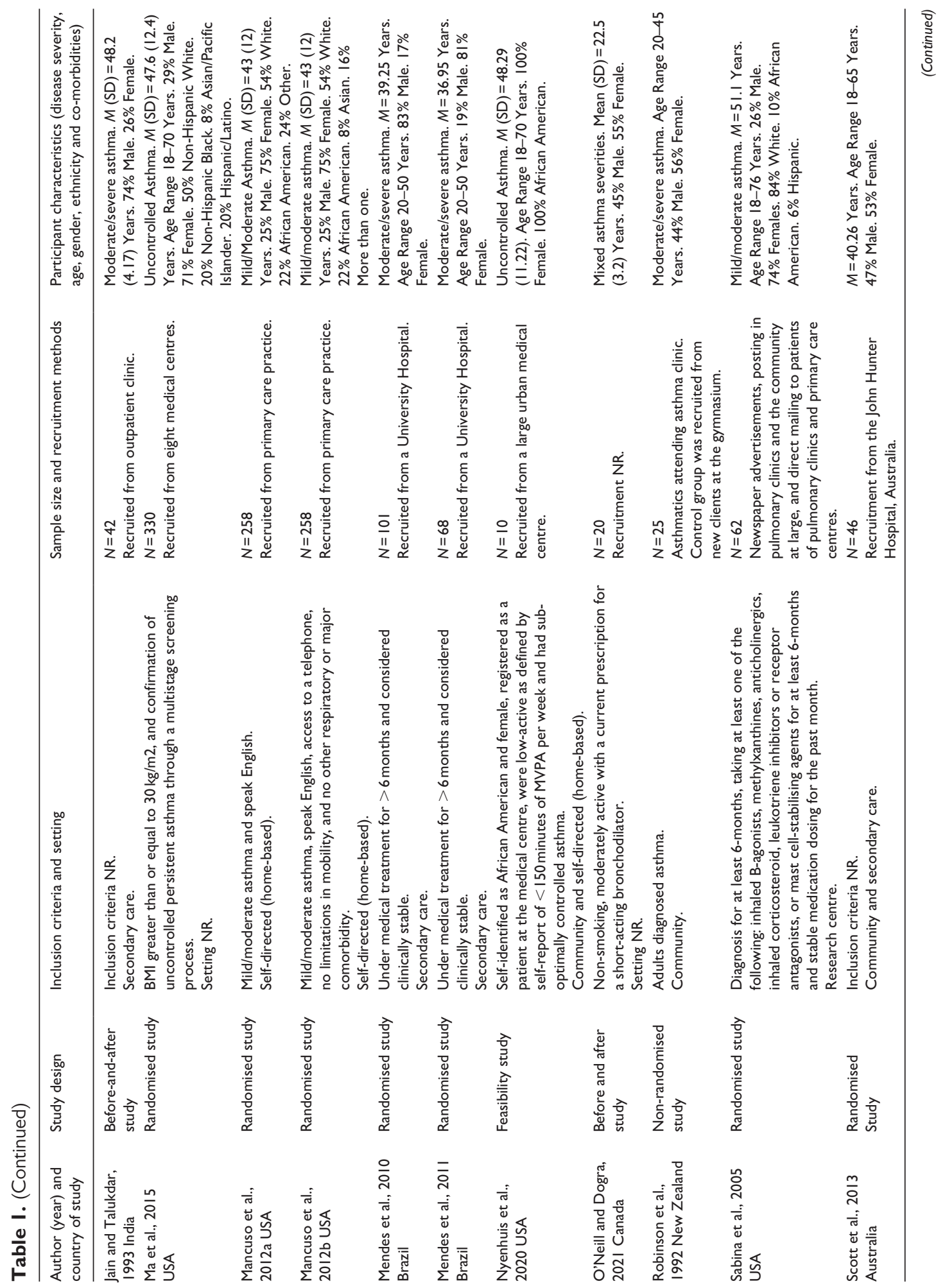




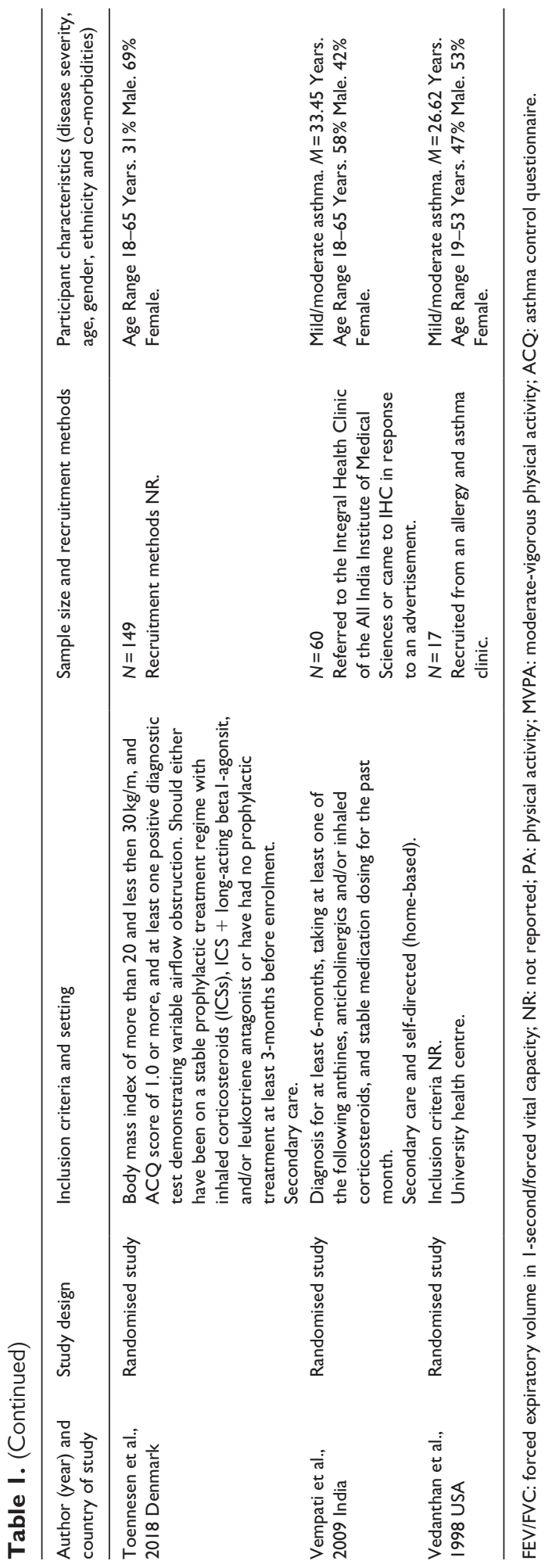

were conducted in either the USA $(n=9)$ or Brazil $(n=8)$, with others in Canada $(n=3)$, India $(n=2)$, New Zealand $(n=1)$, Australia $(n=1)$ and Denmark $(n=1)$. The study designs included randomised controlled trials $(n=18)$, non-randomised controlled trials $(n=2)$, beforeand-after studies $(n=3)$, a quasi-experimental study $(n=1)$ and a feasibility study $(n=1)$. Within these studies, 21 unique interventions were reported.

The number of participants in each study ranged from 10 to 330 . Asthma severity varied across studies, with only two studies failing to report the asthma severity of their participants (Scott et al., 2013; Toennesen et al., 2018). Seven studies included participants with mild/ moderate asthma (Bidwell et al., 2012; Boyd et al., 2012; Mancuso et al., 2012a, 2012b; Sabina et al., 2005; Vedanthan et al., 1998; Vempati et al., 2009), 10 studies included participants with moderate/severe asthma (Coelho et al., 2018; Evaristo et al., 2020; França-Pinto et al., 2015; Freitas et al., 2017, 2018; Goncalves et al., 2008; Jain and Talukdar, 1993; Mendes et al., 2010, 2011; Robinson et al., 1992), and two studies included participants with mixed asthma severity (Hildenbrand et al., 2010; O’Neill and Dogra, 2021). Participants in two studies had partially controlled asthma (Dogra et al., 2010, 2011), and two had uncontrolled asthma (Ma et al., 2015; Nyenhuis et al., 2020).

\section{Risk of bias within studies}

All 25 studies were judged to be at high risk of bias in at least one domain (see Supplementary file 5 and 6 ). The most common sources of bias were blinding of participants (21 studies judged as high risk) and blinding of outcome assessors (12 studies judged as high risk).

\section{Effects of interventions}

We found that ten out of 25 studies, eight unique interventions, reported significant improvements in all the relevant behavioural and/or health outcomes they assessed (Bidwell et al., 2012; Dogra et al., 2011; Freitas et al., 2017; 
Goncalves et al., 2008; Mancuso et al., 2012a; Mendes et al., 2010, 2011; O'Neill and Dogra, 2021; Toennesen et al., 2018; Vempati et al., 2009).

Behavioural outcomes. Intervention effects on behavioural outcomes are presented in Table 2 .

\section{Physical activity}

Ten of the 25 included studies assessed physical activity as an outcome (Coelho et al., 2018; Evaristo et al., 2020; Freitas et al., 2017, 2018; Ma et al., 2015; Mancuso et al., 2012a, 2012b; Nyenhuis et al., 2020; Robinson et al., 1992; Scott et al., 2013), five of which measured the outcome objectively. Four studies found evidence of significant positive between-group effects (Coelho et al., 2018; Freitas et al., 2017, 2018; Ma et al., 2015), and four found significant positive within-group effects (Mancuso et al., 2012a, 2012b; Robinson et al., 1992; Scott et al., 2013). However, in the only study to follow-up participants after the intervention, differences between groups were no longer significant 3-months post-intervention $(p=0.31)$ (Coelho et al., 2018). Although not significant, the remaining two studies did report improvements. Evaristo et al. (2020) found that participants in the intervention and control group increased their daily step count by approximately 2000 steps after the intervention, reaching 10000 steps. Similarly, Nyenhuis et al. (2020) found evidence of a small effect on moderate-to-vigorous physical activity.

\section{Sedentary behaviour}

Sedentary behaviour was only assessed in three out of the 25 included studies (Freitas et al., 2017; Nyenhuis et al., 2020; Scott et al., 2013). Of these, two studies found evidence of a significant within-group decrease in time spent sedentary (Nyenhuis et al., 2020; Scott et al., 2013). Freitas et al. (2017) reported no significant between-group differences in time spent sedentary $(p<0.05)$, but the intervention did not specifically target sedentary behaviour.
Health Outcomes. Intervention effects on health outcomes are presented in Table 3.

\section{Quality of life and asthma control}

Sixteen of the 25 included studies assessed quality of life as an outcome (Bidwell et al., 2012; Coelho et al., 2018; Dogra et al., 2010, 2011; Evaristo et al., 2020; França-Pinto et al., 2015; Freitas et al., 2017; Goncalves et al., 2008; Hildenbrand et al., 2010; Ma et al., 2015; Mancuso et al., 2012b; Mendes et al., 2010; Sabina et al., 2005; Scott et al., 2013; Toennesen et al., 2018; Vempati et al., 2009). Of these, five studies found evidence of a significant positive within-group effect (Dogra et al., 2011; Evaristo et al., 2020; Scott et al., 2013; Toennesen et al., 2018; Vempati et al., 2009) and five found significant positive betweengroup effects (Bidwell et al., 2012; França-Pinto et al., 2015; Freitas et al., 2017; Goncalves et al., 2008; Mendes et al., 2010).

Only twelve studies assessed asthma control as an outcome (Boyd et al., 2012; Coelho et al., 2018; Dogra et al., 2010, 2011; Evaristo et al., 2020; França-Pinto et al., 2015; Freitas et al., 2017; Ma et al., 2015; Mancuso et al., 2012b; O’Neill and Dogra, 2021; Scott et al., 2013; Toennesen et al., 2018). Four reported significant positive within-group effects (Evaristo et al., 2020; Mancuso et al., 2012b; O'Neill and Dogra, 2021; Toennesen et al., 2018), and two reported a significant between-group effect (Dogra et al., 2011; Freitas et al., 2017). Although not significant, several of the remaining studies reported a trend of improved asthma control in the intervention group compared to the control (Boyd et al., 2012; França-Pinto et al., 2015; Ma et al., 2015; Scott et al., 2013). Although Dogra et al. (2010) did not report significant positive effects in asthma control, participants perceived asthma control significantly improved post-intervention ( $p=0.014)$.

\section{Asthma symptoms and medication usage}

Asthma symptoms were assessed as an outcome in eleven studies (Evaristo et al., 2020; 


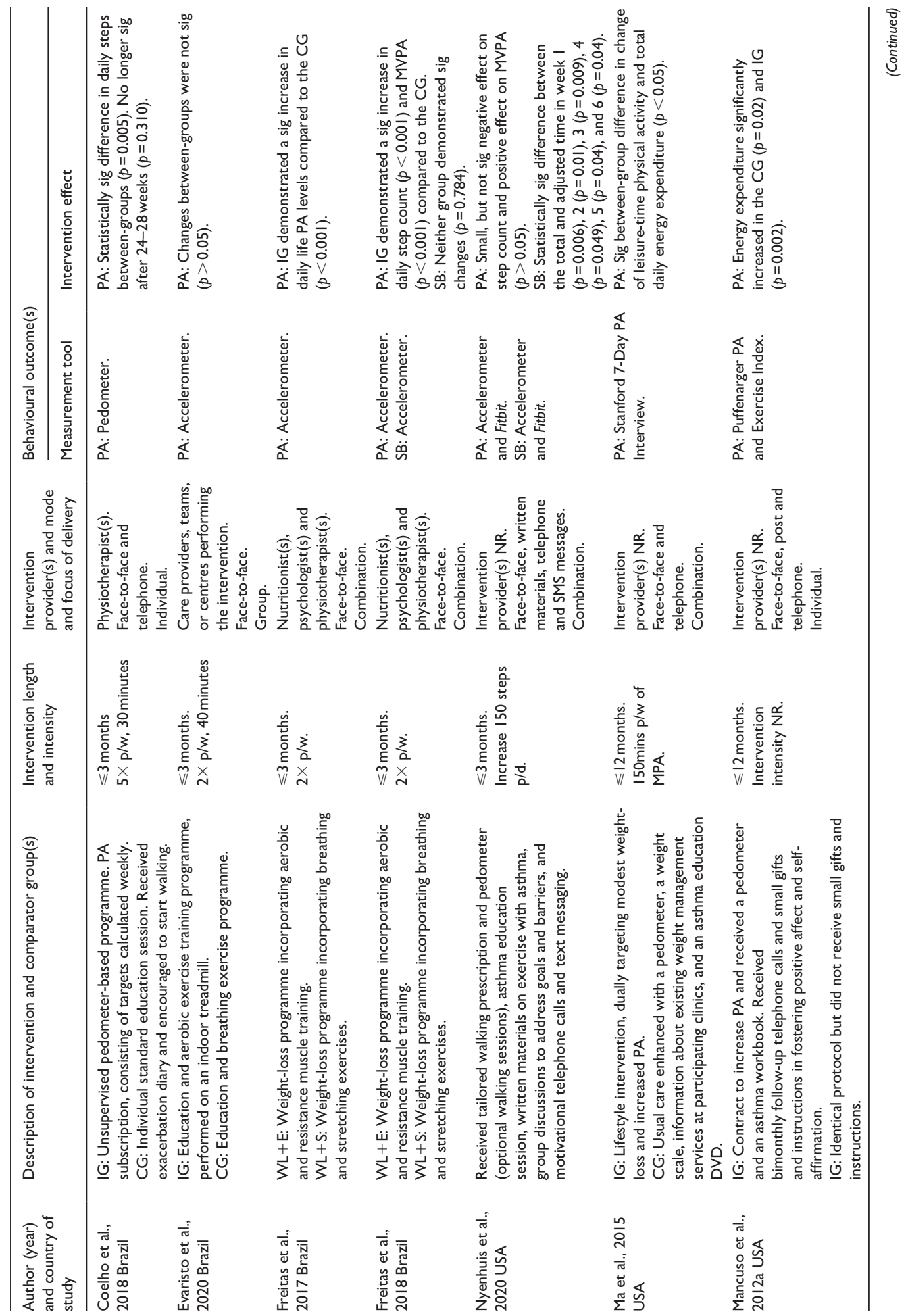




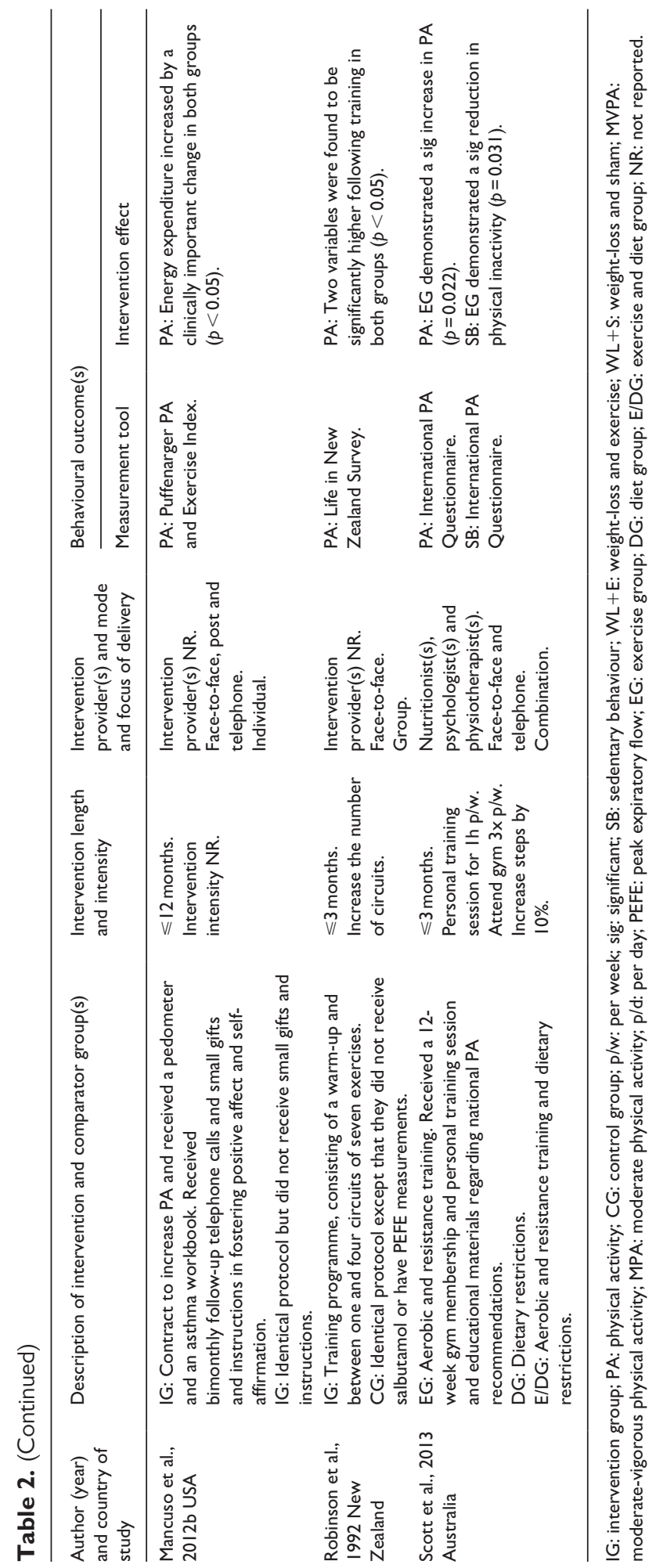




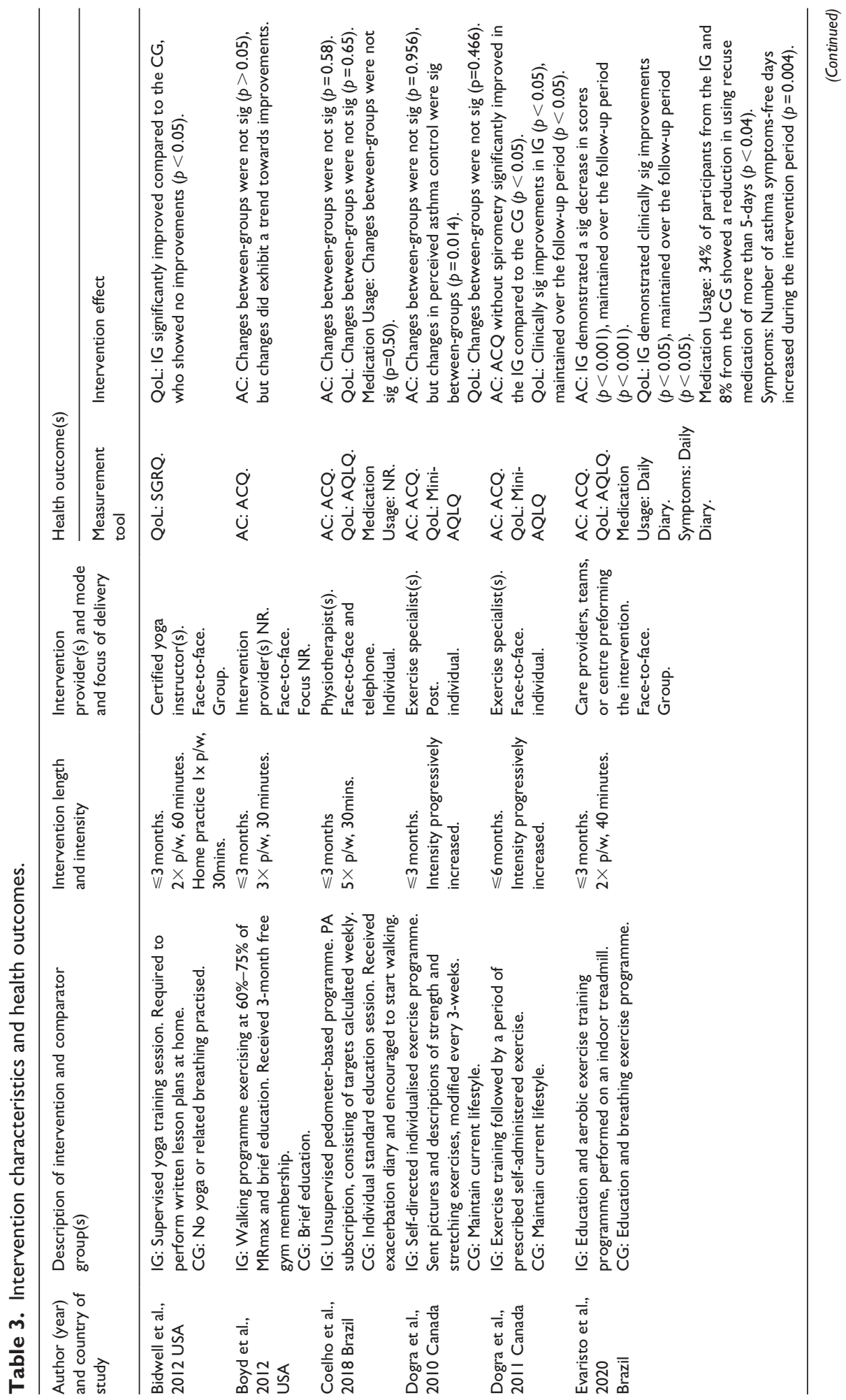




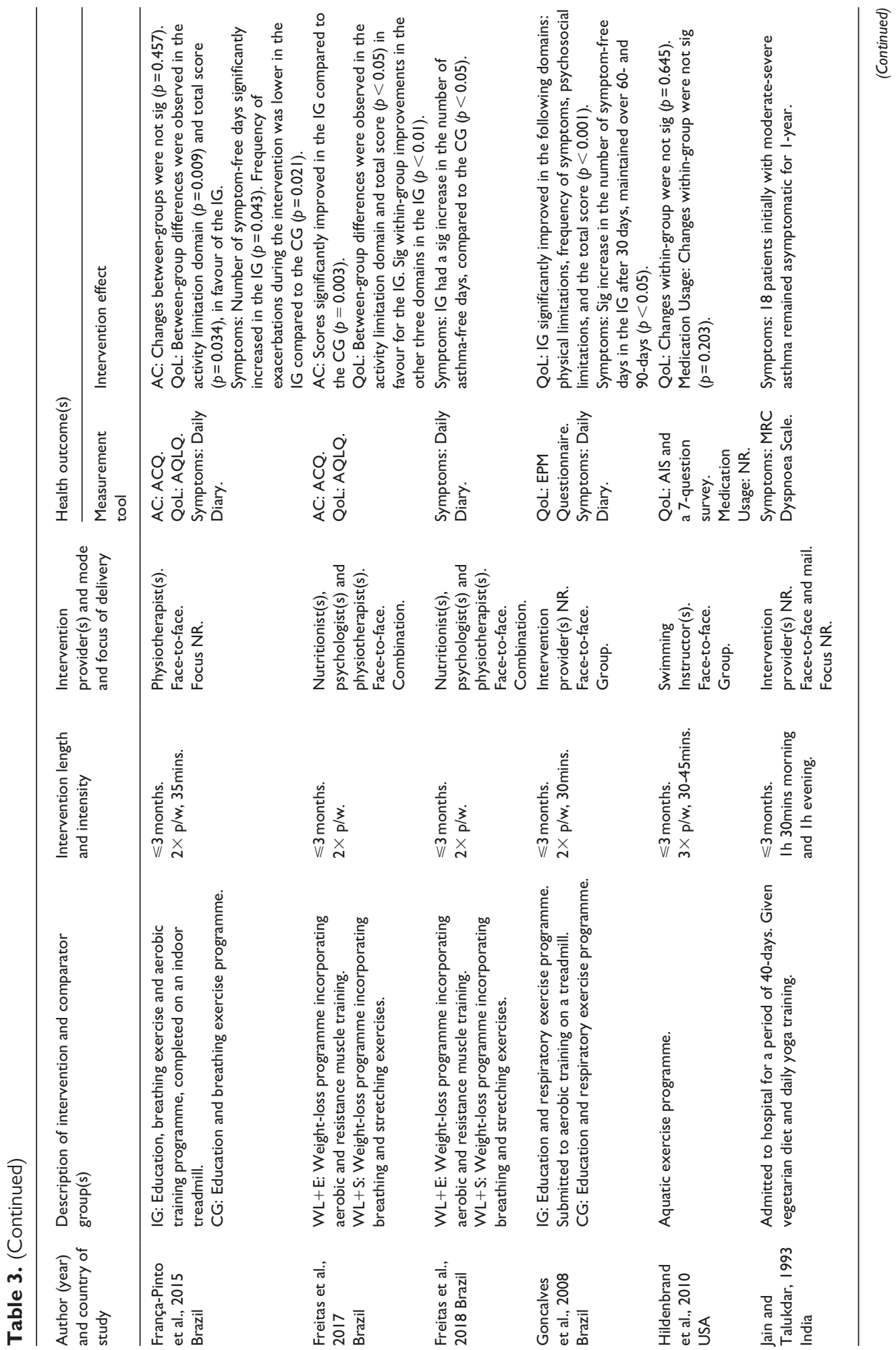




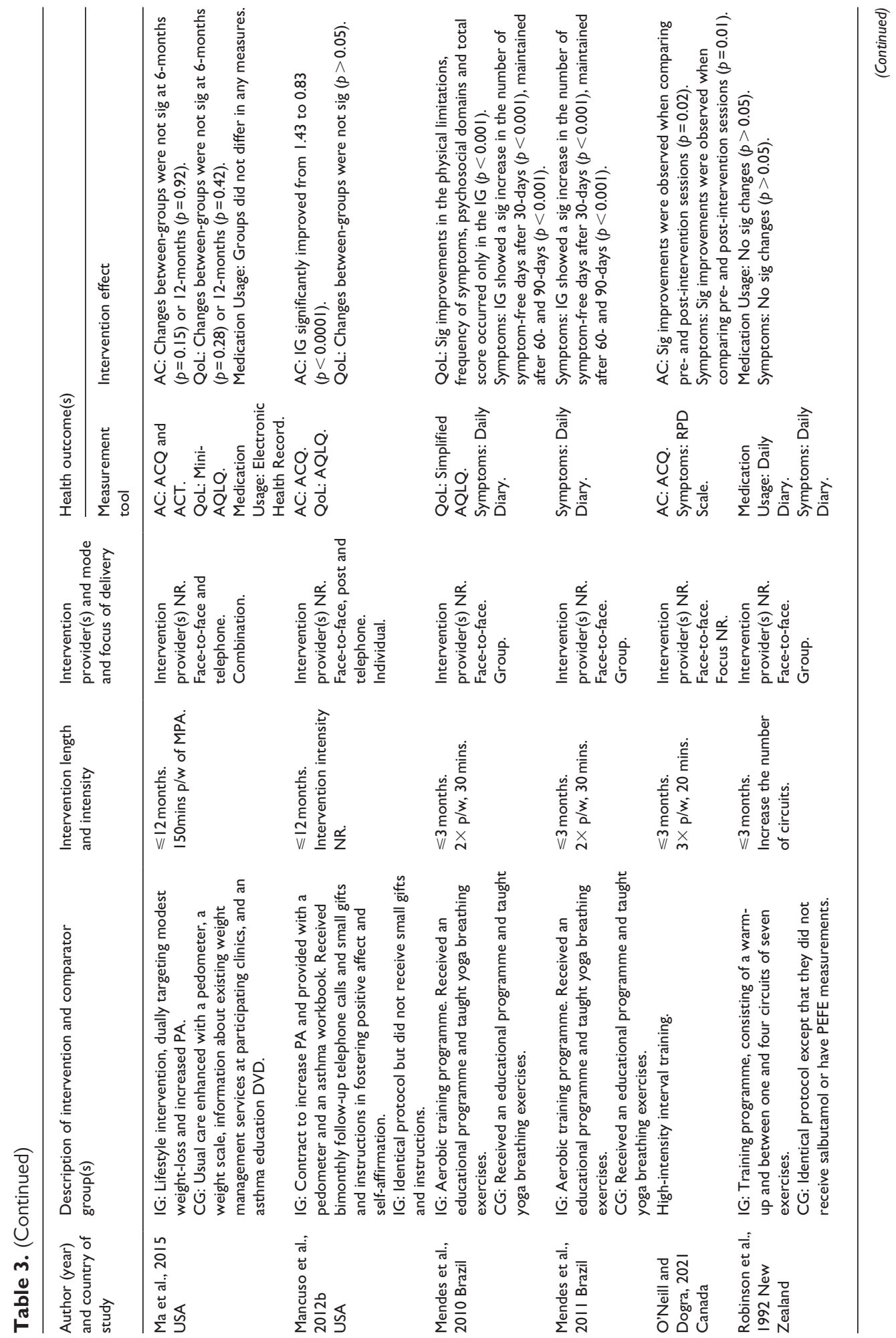




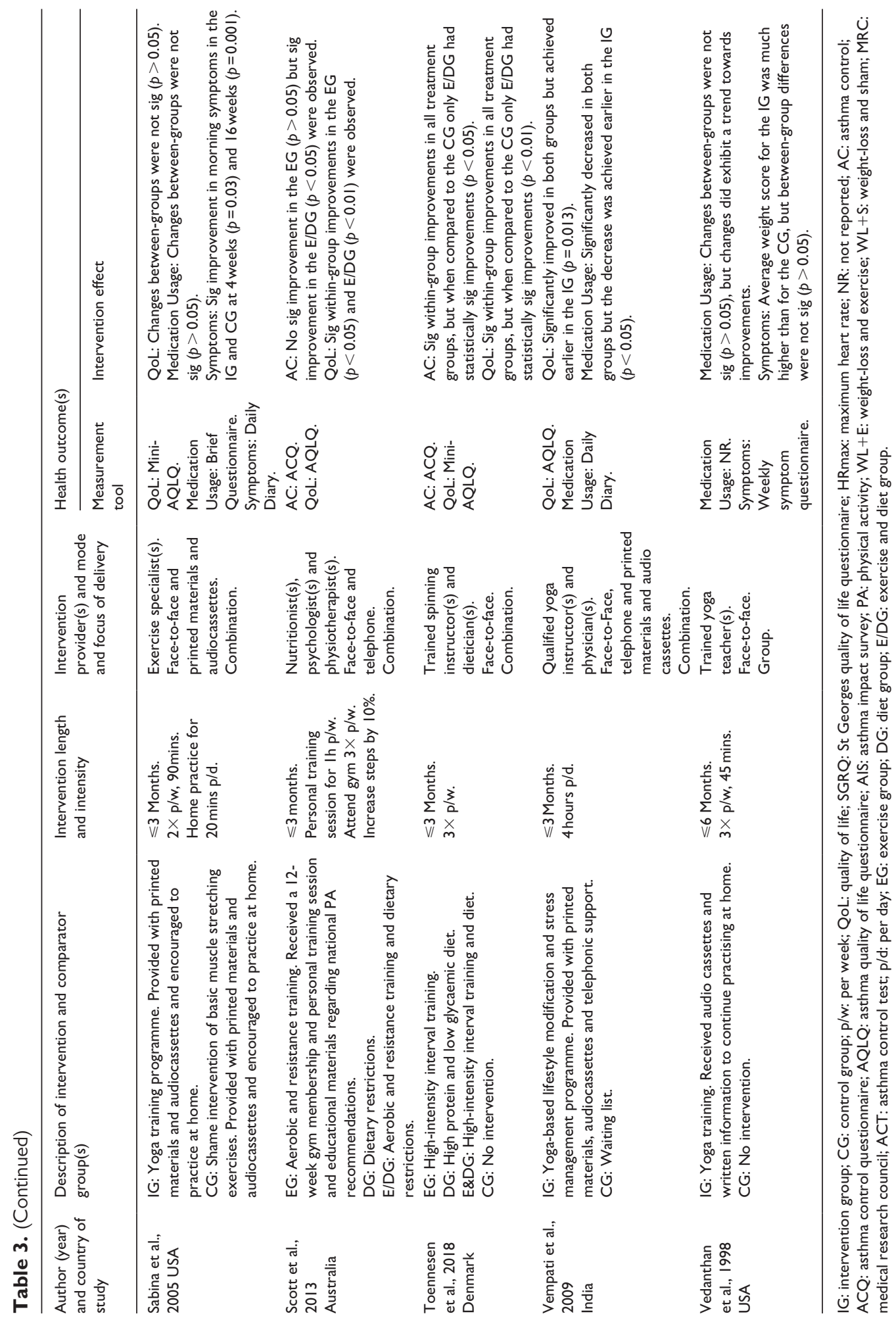


França-Pinto et al., 2015; Freitas et al., 2018; Goncalves et al., 2008; Jain and Talukdar, 1993; Mendes et al., 2010, 2011; O’Neill and Dogra, 2021; Robinson et al., 1992; Sabina et al., 2005; Vedanthan et al., 1998), with four reporting significant positive within-group effects (Evaristo et al., 2020; França-Pinto et al., 2015; O’Neill and Dogra, 2021; Sabina et al., 2005) and four reporting a significant positive between-group effect (Freitas et al., 2018; Goncalves et al., 2008; Mendes et al., 2010, 2011). Although not significant, two of the remaining studies reported improved asthma symptoms in the intervention group over time (Jain and Talukdar, 1993; Vedanthan et al., 1998).

Eight out of the 25 included studies assessed medication usage as an outcome (Coelho et al., 2018; Evaristo et al., 2020; Hildenbrand et al., 2010; Ma et al., 2015; Robinson et al., 1992; Sabina et al., 2005; Vedanthan et al., 1998; Vempati et al., 2009). Only two of the eight studies found within-group evidence of a significant reduction in the use of rescue inhalers in the intervention group (Evaristo et al., 2020; Vempati et al., 2009).

\section{Intervention components}

Interventions were heterogeneous in terms of intervention duration, physical activity type, intensity and duration, mode of delivery, delivery focus and intervention provider. These are presented in Tables 2 and 3.

Intervention duration/intensity and physical activity. The majority of the 21 unique included interventions lasted no more than 3-months $(n=17)$, with others lasting no more than 6-months $(n=2)$ and no more than 12-months $(n=2)$. The activity performed within the intervention varied across studies but included aerobic exercises and/or strength/resistance training $(n=8)$, yoga training $(n=5)$, walking only $(n=5)$, high-intensity interval training $(n=2)$, indoor circuit training $(n=1)$, and aquatic training $(n=1)$. Participants were most commonly asked to perform the activity two or three times per week, for between 30 and 60 minutes $(n=9)$. However, two studies did not report information on the intensity of the intervention (Mancuso et al., 2012a, 2012b).

Intervention provider, mode of delivery and delivery focus. In most interventions, the provider was not reported $(n=8)$, with others using exercise specialists, including yoga and swimming instructors $(n=6)$, physiotherapists $(n=2)$, or a combination of providers $(n=5)$. Intervention providers delivered most of the interventions face-to-face $(n=13)$ or using a combination of methods, including face-toface and telephone, post, printed materials and/or tapes $(n=7)$. Nyenhuis et al. (2020) was the only intervention that used data collected from the participant's activity tracker to send tailored SMS messages. Only one of the included interventions had no face-to-face contact and was delivered via post only (Dogra et al., 2010). The majority of interventions were delivered in groups only $(n=6)$ or used a combination of group and individual sessions $(n=7)$. Four interventions used individual sessions only (Coelho et al., 2018; Dogra et al., 2010, 2011; Mancuso et al., 2012a, 2012b), and four studies did not report the focus of delivery (Boyd et al., 2012; França-Pinto et al., 2015; Jain and Talukdar, 1993; O’Neill and Dogra, 2021).

Relationship between intervention components and outcomes based on a narrative synthesis. Six of the eight unique interventions found to be effective only reported short-term effects ( $<3$ months) (Bidwell et al., 2012; Freitas et al., 2017; Goncalves et al., 2008; Mendes et al., 2010, 2011; O’Neill and Dogra, 2021; Toennesen et al., 2018; Vempati et al., 2009). Participants within these interventions were most commonly asked to perform aerobic exercise and/or strength/resistance training $(n=3)$ two or three times per week for 30-60 minutes $(n=4)$. Most interventions were delivered by a combination of providers $(n=3)$ or did not report the provider $(n=2)$. They were mostly delivered face-to-face $(n=5)$ and within groups $(n=2)$ or 
used a combination of group and individual sessions $(n=3)$.

Behaviour change techniques. A total of 25 of the 93 BCTs from the BCTTv1 were identified (Supplementary File 7). BCTs related to increasing physical activity and, in some studies, reducing sedentary behaviour. The number of BCTs identified in individual interventions ranged from 6 to 15, with an average of 9 BCTs per intervention. The most prevalent BCTs (coded in at least 25\% interventions) were: 'Action Planning' $(n=25)$; 'Goal Setting (Behaviour)' $(n=25)$; 'Instruction on How to Perform Behaviour' $(n=22)$; 'Demonstration of Behaviour' $(n=20)$; 'Behavioural Practice/ Rehearsal' ( $n=20)$; 'Self-Monitoring of Behaviour' ( $n=14)$; 'Self-Monitoring Outcome(s) of Behaviour(s)' $(n=11)$; 'Social Support (Unspecified)' ( $n=10)$; 'Adding Objects to the Environment' $(n=10)$; 'Graded Tasks' $(n=9)$; 'Pharmacological Support' $(n=9)$; 'Monitoring of Outcome(s) of Behaviour Without Feedback' $(n=8)$; 'Body Changes' $(n=8)$; 'Feedback on Behaviour' $(n=7)$; and 'Information About Health Consequences' $(n=7)$. In terms of 'Pharmacological Support', this concerned intervention providers encouraging participants to adhere to their asthma medication. Twenty of the 25 identified BCTs were included in the seven unique, effective interventions. The most commonly used BCTs in effective interventions were: 'Action Planning' (100\%); 'Goal Setting (Behaviour)' (100\%); 'Instruction on how to Perform Behaviour' (89\%); 'Demonstration of Behaviour' (89\%); 'Behavioural Practice/ Rehearsal' (89\%). BCTs identified in effective interventions were also included in the interventions which did not show evidence of effectiveness.

\section{Discussion}

We found that interventions that promote physical activity had significant benefits in terms of increasing physical activity (eight out of ten studies), decreasing time spent sedentary (two out of three studies), improving quality of life (ten out of 16 studies) and decreasing asthma symptoms (eight out of 11 studies). However, we found no evidence of a positive effect on asthma control (six out of 12 studies) and medication usage (two out of eight studies). Ten out of the 25 included studies, comprising 786 out of 1849 participants, reported significant positive effects in all the relevant outcomes they assessed and were deemed effective. Participants within these interventions were most commonly asked to perform aerobic exercise and/or strength/resistance training two or three times per week for 30-60 minutes. Most interventions were delivered by a combination of providers or did not report the provider. They were delivered face-to-face and within groups or used a combination of group and individual sessions. However, we cannot say for definite that these intervention components increased effectiveness as components were similar across all interventions regardless of their effectiveness. The most commonly used BCTs in effective interventions were: 'Action Planning' (100\%); 'Goal Setting' (100\%); 'Instruction on how to Perform the Behaviour' (89\%); 'Demonstration of Behaviour' (89\%); and 'Behavioural Practice/Rehearsal' (89\%). Due to the similarities of the BCTs used across all intervention and control groups, it was not possible to identify specific BCTs that showed promise of effectiveness.

Notably absent from the BCTs extracted from the included interventions were techniques that help self-regulate behaviour and sustain motivation, which are important for adopting and maintaining behaviour change. For example, 'Problem Solving', 'Reviewing Behavioural Goals', 'Prompts/Cues', 'Habit Formation' and 'Self Reward' have all been associated with behaviour maintenance (Howlett et al., 2019; Samdal et al., 2017), but were not identified in the included interventions. Although there was an increase in physical activity during the intervention period, the only intervention to follow-up participants beyond the intervention showed that the increase was not maintained (Coelho et al., 2018). For changes to be maintained, future 
interventions should consider including the above techniques.

In terms of active ingredients of the intervention, 'Goal Setting' and 'Action Planning' were the most commonly used BCTs. Metaanalyses have shown that setting a specific and detailed plan on when, where and how to perform a behaviour and providing instructions increases self-efficacy (one's belief in their ability to engage in the behaviour successfully) and physical activity (Williams and French, 2011). Qualitative studies have found that major barriers to physical activity for adults with asthma are low self-efficacy and negative beliefs about their capabilities to be active (Mancuso et al., 2006; Nyenhuis et al., 2019). This would suggest that these BCTs are important for behaviour change in this population group, and although we cannot conclude that these are 'promising' or more likely to increase effectiveness as they were identified in both effective and ineffective interventions, the evidence supporting the use of these BCTs is well established.

Although identified in a small number of interventions, some evidence-based BCTs could be used more often to increase the likelihood of interventions being effective. For example, incorporating 'Practical Social Support' has been associated with better intervention effects. Planning how to elicit social support from individuals makes participants feel more in control and ensures that they are supported to make behavioural changes (Olander et al., 2013), but this BCT was only identified in one of the included studies. Social support has been reported as a facilitator to physical activity in this patient group (Mancuso et al., 2006; Nyenhuis et al., 2019). Including this BCT in future interventions could increase their effectiveness by ensuring that participants are supported and could help them cope with possible setbacks following asthma flare-ups. Another BCT associated with increased physical activity is 'Self-Monitoring of Outcomes of Behaviour' (Olander et al., 2013). One of the major barriers to physical activity reported by adults with asthma, even for those with mild asthma, is fear and anxiety of triggering symptoms (Clarke and Mansur, 2015). If participants could see the benefits of physical activity to their asthma outcomes for themselves, it could reduce negative feelings and encourage them to continue to make positive improvements to increase their activity levels. Other BCTs that reduce negative emotions and conserve mental resources and BCTs in the 'Self Belief' group, such as 'Verbal Persuasion About Capability' and 'Focus on Past Success', could be included.

As there are limited facilities and funding available to deliver PR to patients, alternative interventions to PR could increase the number of patients who can access help and support to improve their physical activity levels. However, although the included interventions were not PR, most of them would not have overcome the major barriers previously reported by patients (Jones et al., 2017; Keating et al., 2011) as they still required participants to travel and would not have been suitable for those living with comorbidities. These barriers need to be considered in the development of future physical activity interventions, and alternative delivery methods should be considered to provide patients with tailored interventions at a convenient time and place for them. To help overcome these barriers, home-based programmes have been proposed as an alternative to traditional PR. Randomised controlled trials have shown that it produced short-term clinical outcomes equivalent to traditional PR (Holland et al., 2017), including improving exercise capacity and quality of life (Pradella et al., 2015). Homebased PR could be optimised by incorporating the BCTs most commonly used in effective interventions highlighted in this review to increase the number of people who can access help and support and improve long-term effects. Our findings highlight the potential use of digital interventions that have unique advantages over traditional in-person interventions, being more accessible and convenient for participants (Griffiths et al., 2006). Except for Nyenhuis et al. (2020), none of the included interventions were digital or had a digital component. Future research should investigate the development of 
digital-physical activity interventions tailored to adults with asthma, and good-quality randomised trials should be carried out to understand their effectiveness in this population group.

\section{Strengths and limitations}

This review followed a structured search protocol that used five electronic databases. The inclusion criteria were broad, and we included a wide range of study designs and interventions with all asthma severities. We used a detailed categorisation of BCTs with reference to standard classifications. However, some limitations need to be considered. There was a great deal of heterogeneity in the included studies in relation to the research design, type of intervention and outcome data. Therefore, we could not conduct a meta-analysis, and only a narrative review could be produced.

Furthermore, the level of detail needed for extracting BCTs was often not present in published intervention descriptions; included interventions might have used more BCTs than those extracted in this review. We addressed this problem by having two reviewers independently extract BCTs, and we included both probably $($ coded + ) and definite $($ coded ++ ) BCTs. In addition to this, it was not always clear whether an intervention would qualify as PR or not due to the lack of detail provided in the intervention descriptions. Again, we tried to address this problem by a second reviewer validating the inclusion and exclusion of papers with the option to discuss with a third reviewer if there was a discrepancy. Nevertheless, this review provides an update on the literature on physical activity interventions and their effects on asthma outcomes and provides an insight into the literature gaps that need to be addressed in future studies.

\section{Conclusion}

We included 25 studies that reported 21 unique physical activity interventions within this review. Interventions significantly increased physical activity levels and decreased sedentary behaviour in adults diagnosed with asthma. They also positively affected quality of life and asthma symptoms, but not asthma control and medication usage. Ten out of the 25 included studies reported significant positive effects in all the relevant outcomes they assessed and were deemed effective. Participants within these interventions were most commonly asked to perform aerobic exercise and/or strength/resistance training two or three times per week for 30-60 minutes. Most interventions were delivered by a combination of providers or did not report the provider. They were delivered face-to-face and within groups or used a combination of group and individual sessions. The most commonly used BCTs in effective interventions were: 'Action Planning'; 'Goal Setting (Behaviour)'; 'Instruction on how to Perform the Behaviour'; 'Demonstration of the Behaviour'; and 'Behavioural Practice/ Rehearsal'. Due to the similarities in components and BCTs used across all intervention and control groups, it was not possible to identify specific intervention components that showed promise of effectiveness. We recommend that future interventions include evidence-based techniques that prompt self-regulation of behaviour and sustain motivation and behaviour change.

\section{Declaration of conflicting interests}

The author(s) declared no potential conflicts of interest with respect to the research, authorship, and/or publication of this article.

\section{Funding}

The author(s) disclosed receipt of the following financial support for the research, authorship, and/or publication of this article: This study was conducted as part of the author Leanne Tyson's PhD project, which has been funded by Asthma UK Centre for Applied Research and the University of East Anglia Faculty of Medicine and Health Sciences [Reference Number: 10025744].

\section{ORCID iD}

Leanne Tyson (iD) https://orcid.org/0000-0001-7119 $-1535$ 


\section{Supplemental material}

Supplemental material for this article is available online.

\section{References}

*Indicates studies included in the review.

*Bidwell AJ, Yazel B, Davin D, et al. (2012) Yoga training improves quality of life in women with asthma. Journal of Alternative and Complementary Medicine 18(8): 749-755.

*Boyd A, Yang CT, Estell K, et al. (2012) Feasibility of exercising adults with asthma: A randomized pilot study. Allergy Asthma and Clinical Immunology 8(1): 13.

British Thoracic Society (2019) SIGN 158 guidelines on the management of asthma: A national clinical guidelines. Available at: https:/www. brit-thoracic.org.uk/quality-improvement/ guidelines/asthma/ (accessed 4th May 2021).

Clarke A and Mansur A (2015) P248 Self-reported activity levels, barriers and facilitators to exercise in severe asthma. Thorax 70(Suppl 3): A201.2-A202.

*Coelho CM, Reboredo MM, Valle FM, et al. (2018) Effects of an unsupervised pedometerbased physical activity program on daily steps of adults with moderate to severe asthma: A randomized controlled trial. Journal of Sports Sciences 36(10): 1186-1193.

Cordova-Rivera L, Gibson PG, Gardiner PA, et al. (2018) A systematic review of associations of physical activity and sedentary time with asthma outcomes. The Journal of Allergy and Clinical Immunology in Practice 6(6): 1968-1981.e2.

*Dogra S, Jamnik V and Baker J (2010) Self-directed exercise improves perceived measures of health in adults with partly controlled asthma. Journal of Asthma 47(9): 972-977.

*Dogra S, Kuk JL, Baker J, et al. (2011) Exercise is associated with improved asthma control in adults. European Respiratory Journal 37: 318-323.

*Evaristo KB, Mendes FAR, Saccomani MG, et al. (2020) Effects of aerobic training versus breathing exercises on asthma control: A randomized trial. The Journal of Allergy and Clinical Immunology in Practice 8(9): 2989-2996.e4.

Feng Z, Wang J, Xie Y, et al. (2021) Effects of exercise-based pulmonary rehabilitation on adults with asthma: A systematic review and metaanalysis. Respiratory Research 22(1): 33.
*França-Pinto A, Mendes FA, de Carvalho-Pinto RM, et al. (2015) Aerobic training decreases bronchial hyperresponsiveness and systemic inflammation In patients with moderate or severe asthma: A randomised controlled trial. Thorax 70(8): 732-739.

*Freitas PD, Ferreira PG, Silva AG, et al. (2017) The role of exercise in a weight-loss program on clinical control in obese adults with asthma. A randomized controlled trial. American Journal of Respiratory and Critical Care Medicine 195(1): 32-42.

*Freitas PD, Silva AG, Ferreira PG, et al. (2018) Exercise improves physical activity and comorbidities in obese adults with asthma. Medicine and Science in Sports and Exercise 50(7): 1367-1376.

Global Initiative for Asthma (2018) Global strategy for asthma management and prevention. Available at: https://ginasthma.org/wp-content/ uploads/2018/04/wms-GINA-2018-reportV1.3-002.pdf (accessed 4th May 2021).

*Goncalves RC, Nunes MPT, Cukier A, et al. (2008) Effects of an aerobic physical training program on psychosocial characteristics, quality-of-life, symptoms and exhaled nitric oxid in individuals with moderate or severe persistent asthma. Brazilian Journal of Physical Therapy 12(2): 127-135.

Griffiths F, Lindenmeyer A, Powell J, et al. (2006) Why are health care interventions delivered over the internet? A systematic review of the published literature. Journal of Medical Internet Research 8(2): e10.

Hansen ESH, Pitzner-Fabricius A, Toennesen LL, et al. (2020) Effect of aerobic exercise training on asthma in adults: AA systematic review and meta-analysis. European Respiratory Journal 56(1): 2000146.

Hayton C, Clark A, Olive S, et al. (2013) Barriers to pulmonary rehabilitation: Characteristics that predict patient attendance and adherence. Respiratory Medicine 107(3): 401-407.

Higgins JPT, Thomas J, Chandler M, et al. (2021) Cochrane handbook for systematic reviews of interventions version 6.2 (updated February 2021). Available at: https://training.cochrane. org/handbook/current (accessed 4th May 2021).

*Hildenbrand K, Nordio S, Freson TS, et al. (2010) Development of an aquatic exercise training protocol for the asthmatic population. International 
Journal of Aquatic Research and Education 4(3): 278-299. DOI: 10.25035/ijare.04.03.07

Holland AE, Mahal A, Hill CJ, et al. (2017) HomeBased rehabilitation for COPD using minimal resources: A randomised, controlled equivalence trial. Thorax 72(1): 57-65.

Howlett N, Trivedi D, Troop NA, et al. (2019) Are physical activity interventions for healthy inactive adults effective in promoting behavior change and maintenance, and which behavior change techniques are effective? A systematic review and meta-analysis. Translational Behavioral Medicine 9(1): 147-157.

Jácome C and Marques A (2014) Impact of pulmonary rehabilitation in subjects with mild COPD. Respiratory Care 59(10): 1577-1582.

*Jain SC and Talukdar B (1993) Evaluation of yoga therapy programme for patients of bronchial asthma. Singapore Medical Journal 34: 306308.

Jones AW, Taylor A, Gowler H, et al. (2017) Systematic review of interventions to improve patient uptake and completion of pulmonary rehabilitation in COPD. ERJ Open Research 3(1): 00089-02016.

Keating A, Lee A and Holland AE (2011) What prevents people with chronic obstructive pulmonary disease from attending pulmonary rehabilitation? A systematic review. Chronic Respiratory Disease 8(2): 89-99.

Linhas R, Marçôa R, Ladeira I, et al. (2017) Effects of pulmonary rehabilitation in asthma patients. European Respiratory Journal 50: PA757. DOI: 10.1183/1393003.congress.2017.PA757

* Ma J, Strub P, Xiao L, et al. (2015) Behavioral weight loss and physical activity intervention in obese adults with asthma. A randomized trial. Annals of the American Thoracic Society 12(1): $1-11$.

*Mancuso CA, Choi TN, Westermann $\mathrm{H}$, et al. (2012a) Increasing physical activity in patients with asthma through positive affect and selfaffirmation: A randomized trial. Archive of Internal Medicine 172(4): 337-343.

*Mancuso CA, Choi TN, Westermann H, et al. (2012b) Improvement in asthma quality of life in patients enrolled in a prospective study to increase lifestyle physical activity. Journal of Asthma 50(1): 103-107.

Mancuso CA, Sayles W, Robbins L, et al. (2006) Barriers and facilitators to healthy physical activity in asthma patients. Journal of Asthma 43(2): 137-143.
McCarthy B, Casey D, Devane D, et al. (2015) Pulmonary rehabilitation for chronic obstructive pulmonary disease. Cochrane Database of Systematic Reviews 23(2): CD003793.

*Mendes FA, Almeida FM, Cukier A, et al. (2011) Effects of aerobic training on airway inflammation in asthmatic patients. Medicine and Science in Sports and Exercise 43(2): 197-203.

*Mendes FA, Gonçalves RC, Nunes MP, et al. (2010) Effects of aerobic training on psychosocial morbidity and symptoms in patients with asthma: A randomized clinical trial. Chest 138(2): 331-337.

Michie S, Richardson M, Johnston M, et al. (2013) The behavior change technique taxonomy (v1) of 93 hierarchically clustered techniques: Building an international consensus for the reporting of behavior change interventions. Annals of Behavioral Medicine 46(1): 81-95.

Moher D, Liberati A, Tetzlaff J, et al. (2009) Preferred reporting items for systematic reviews and meta-analyses: The PRISMA statement. BMJ 339: b2535.

National COPD Audit Programme (2015) Pulmonary rehabilitation: Time to breathe better. Available at: https://www.rcplondon.ac.uk/projects/outputs/pulmonary-rehabilitation-time-breathebetter (accessed 4th May 2021).

*Nyenhuis SM, Balbim GM, Ma J, et al. (2020) A walking intervention supplemented with mobile health technology in low-active urban African American women with asthma: proof-of-concept study. JMIR Formative Research 4(3): e13900.

Nyenhuis SM, Shah N, Ma J, et al. (2019) Identifying barriers to physical activity among African American women with asthma. Cogent Medicine 6: 1.

Olander EK, Fletcher H, Williams S, et al. (2013) What are the most effective techniques in changing obese individuals' physical activity self-efficacy and behaviour: A systematic review and meta-analysis. International Journal of Behavioral Nutrition and Physical Activity 10(1): 29.

*O'Neill C and Dogra S (2021) Low volume high intensity interval training leads to improved asthma control in adults. Journal of Asthma 58: 1256-1260.

Pradella CO, Belmonte GM, Maia MN, et al. (2015) Home-Based pulmonary rehabilitation for subjects with COPD: A randomized study. Respiratory Care 60(4): 526-532. 
*Robinson DM, Egglestone DM, Hill PM, et al. (1992) Effects of a physical conditioning programme on asthmatic patients. The New Zealand Medical Journal 105(937): 253-256.

*Sabina AB, Williams A, Wall HK, et al. (2005) Yoga intervention for adults with mild-to-moderate asthma: A pilot study. Annals of Allergy Asthma \& Immunology 94(5): 543-548.

Samdal GB, Eide GE, Barth T, et al. (2017) Effective behaviour change techniques for physical activity and healthy eating in overweight and obese adults: Systematic review and meta-regression analyses. International Journal of Behavioral Nutrition and Physical Activity 14(1): 42. DOI: 10.1186/s12966-017-0494-y

*Scott HA, Gibson PG, Garg ML, et al. (2013) Dietary restriction and exercise improve airway inflammation and clinical outcomes in overweight and obese asthma: A randomized trial. Clinical \& Experimental Allergy 43(1): 36-49.

Spruit MA, Singh SJ, Garvey C, et al. (2013) An official American Thoracic Society/European Respiratory Society statement: Key concepts and advances in pulmonary rehabilitation. American Journal of Respiratory and Critical Care Medicine 188(8): e13-e64.
*Toennesen LL, Meteran H, Hostrup M, et al. (2018) Effects of exercise and diet in nonobese asthma patients-A randomized controlled trial. The Journal of Allergy and Clinical Immunology In Practice 6(3): 803-811.

van 't Hul AJ, Frouws S, van Den Akker E, et al. (2016) Decreased physical activity in adults with bronchial asthma. Journal of Respiratory Medicine 114: 72-77.

*Vedanthan PK, Kesavalu LN, Murthy KC, et al. (1998) Clinical study of yoga techniques in university students with asthma: A controlled study. Allergy and Asthma Proceedings 19(1): 3-9.

*Vempati R, Bijlani RL and Deepak KK (2009) The efficacy of a comprehensive lifestyle modification programme based on yoga in the management of bronchial asthma: A randomized controlled trial. BMC Pulmonary Medicine 9: 37.

Williams SL and French DP (2011) What are the most effective intervention techniques for changing physical activity self-efficacy and physical activity behaviour-and are they the same? Health Education Research 26(2): 308-322. 